DESY $02-100$

Edinburgh 2002/05

LU-ITP 2002/014

\title{
A lattice study of the spin structure of the $\Lambda$ hyperon
}

\author{
M. Göckeler ${ }^{\mathrm{a}, \mathrm{b}}$, R. Horsley ${ }^{\mathrm{c}}$, D. Pleiter ${ }^{\mathrm{d}}$, P.E.L. Rakow ${ }^{\mathrm{b}}$, S. Schaefer ${ }^{\mathrm{b}}$, \\ A. Schäfer ${ }^{\mathrm{b}}$, and G. Schierholz ${ }^{\mathrm{d}, \mathrm{e}}$ \\ - QCDSF Collaboration - \\ a Institut für Theoretische Physik, Universität Leipzig, \\ D-04109 Leipzig, Germany, \\ b Institut für Theoretische Physik, Universität Regensburg, \\ D-93040 Regensburg, Germany, \\ ${ }^{c}$ Department of Physics and Astronomy, University of Edinburgh, \\ Edinburgh EH9 3JZ, Scotland, UK \\ d John von Neumann-Institut für Computing NIC, \\ D-15735 Zeuthen, Germany \\ ${ }^{\text {e }}$ Deutsches Elektronen-Synchrotron DESY, D-22603 Hamburg, Germany
}

\begin{abstract}
:
The internal spin structure of the $\Lambda$ is of special importance for the understanding of the spin structure of hadrons in general. The comparison between the nucleon and $\Lambda$ allows for a test of the relevant flavour-symmetry breaking effects. Using nonperturbatively $O(a)$ improved Wilson fermions in the quenched approximation we have calculated the first moments of the unpolarised, longitudinally polarised and transversity quark distribution functions in the $\Lambda$. The results indicate that flavour symmetry breaking has little effect on the internal spin structure, in accordance with model based expectations.
\end{abstract}

PACS: 11.15.Ha, 11.30.Hv, 12.38.Gc, 14.20.Jn 


\section{INTRODUCTION}

The detailed investigation of the nucleon spin structure during the last few years has fuelled a large number of efforts to understand the results with quark models of various kinds. It turned out during the course of these investigations that $\mathrm{SU}(3)$ flavour breaking is of central importance for any such effort. A small selection of relevant articles can be found in [1]. Presently the different predictions cover a rather broad range, and while some authors claim great accuracy, others conclude that the existing experimental data on hyperon decays is insufficient for any stringent prediction [2]. The $\Lambda$ spin structure, in turn, is especially sensitive to flavour $\mathrm{SU}(3)$ breaking [3, , 4, 5]. While in the naive $\mathrm{SU}(6)$ quark model the spin of the $\Lambda$ is carried exclusively by the $s$ quarks, the $\mathrm{SU}(3)$ rotated results for the nucleon spin structure suggest that the $s$ and $\bar{s}$ quarks carry only $\approx 60 \%$ of the $\Lambda$ spin while $u, \bar{u}$, $d$ and $\bar{d}$ quarks quarks contribute $\approx-40 \%$. Any specific assumptions about $\mathrm{SU}(3)$ symmetry breaking will strongly affect these conclusions. Obviously additional input is needed, some of which we want to provide with the lattice results presented in this paper.

The spin structure of the $\Lambda$ is also experimentally of special interest, because its polarisation can easily be measured via the self-analysing weak decay $\Lambda \rightarrow p \pi^{-}$. Indeed, the $\Lambda$ polarisation has been determined at the $Z^{0}$ pole in $e^{+} e^{-}$annihilation [6] where it is mainly due to the $s$ quarks, which when produced via $Z^{0}$ decays have an average polarisation of -0.91 . Furthermore, $\Lambda$ polarisation has been studied in deep-inelastic scattering of polarised positrons on unpolarised protons in the current fragmentation region, i.e. selecting $\Lambda$ s which most likely originate from the struck quark [7]. Needless to say, however, that the interpretation of the experiments is complicated by fragmentation effects, $\Lambda$ s from the decay of heavier hyperons, etc. $\Lambda$ and $\bar{\Lambda}$ polarisation was also analysed for charged-current neutrino nucleon reactions [8]. A completely unsettled puzzle is the very strong polarisation of $\Lambda$ s produced in unpolarised $p+N$ reactions [9]. The knowledge of the internal spin structure of the $\Lambda$ is certainly a necessary ingredient for any explanation.

Thus, there is ample motivation to perform lattice calculations which provide information on the internal $\Lambda$ structure. For a spin $1 / 2$ baryon the fraction $\Delta q$ of the spin carried by the quarks (and antiquarks) of flavour $q$ is given in 
terms of the forward matrix element of the axial vector current:

$$
\left\langle p, s\left|\bar{q} \gamma_{\mu} \gamma_{5} q\right| p, s\right\rangle=2 s_{\mu} \Delta q,
$$

where $s^{2}=-M^{2}(M=$ mass of the baryon) and the states are normalised according to $\left\langle p, s \mid p^{\prime}, s^{\prime}\right\rangle=2 E_{p}(2 \pi)^{3} \delta\left(\vec{p}-\vec{p}^{\prime}\right) \delta_{s s^{\prime}}$. Simultaneously we have calculated the transversity matrix element (the tensor charges $\delta q$ ):

$$
\left\langle p, s\left|\bar{q} \mathrm{i} \sigma_{\mu \nu} \gamma_{5} q\right| p, s\right\rangle=\frac{2}{M}\left(s_{\mu} p_{\nu}-s_{\nu} p_{\mu}\right) \delta q .
$$

The physical origin of the difference between $\Delta q$ and $\delta q$ is that in the first case the baryon is boosted along its spin direction and in the second case perpendicular to it. Thus this difference has a very specific sensitivity to details of the internal baryon structure, being e.g. zero in the non-relativistic limit. Operators with additional derivatives can be used to extract also some higher moments of the corresponding distribution functions.

Our aim is to study how strongly the mass difference between the strange quark and the light quarks affects the flavour SU(3) symmetry between the (polarised) distribution functions in the nucleons and the $\Lambda$. Perfect SU(3) symmetry predicts the equations

$$
\begin{aligned}
\Delta s_{\Lambda} & =\left(2 \Delta u_{p}-\Delta d_{p}+2 \Delta s_{p}\right) / 3 \\
\Delta d_{\Lambda}=\Delta u_{\Lambda} & =\left(\Delta u_{p}+4 \Delta d_{p}+\Delta s_{p}\right) / 6
\end{aligned}
$$

and the same relations for the tensor charges.

A preliminary account of some of our results has already been given in Ref. [10.

\section{THE SIMULATION}

We have performed quenched simulations with the Wilson gauge action at $\beta=6.0$ using nonperturbatively $O(a)$ improved fermions (clover fermions) with $c_{\mathrm{SW}}=1.769$. The lattice size was $16^{3} \times 32$. We worked with nine combinations of hopping parameters: $\kappa_{u}=\kappa_{d}, \kappa_{s} \in\{0.1324,0.1333,0.1342\}$ corresponding to bare quark masses of $\approx 166,112,58 \mathrm{MeV}$, respectively. So we can extrapolate to the chiral limit in $\kappa_{u}=\kappa_{d}$ and interpolate in $\kappa_{s}$ to the physical value $\kappa_{s}^{*}$. For the critical hopping parameter $\kappa_{c}$ we take the value 0.135201 determined from the PCAC quark mass [1], $\kappa_{s}^{*}=0.1341$ was fixed 
by requiring the pseudoscalar mass $m_{\mathrm{PS}}$ for $\kappa_{u}=\kappa_{d}=\kappa_{c}$ and $\kappa_{s}=\kappa_{s}^{*}$ to be equal to the $K^{+}$mass of $494 \mathrm{MeV}$ (with the scale set by the force parameter $r_{0}=0.5 \mathrm{fm}$ ). In the extra- and interpolation we assumed a linear dependence of $m_{\mathrm{PS}}^{2}$ on the quark mass.

As an interpolating field for the $\Lambda$ we used (employing Euclidean notation from now on)

$$
\Lambda_{\alpha}(t)=\sum_{x, x_{4}=t} \epsilon_{i j k} s_{i \alpha}(x)\left(u_{j}^{T}(x) C \gamma_{5} d_{k}(x)\right)
$$

where $C$ is the charge conjugation matrix; $i, j$, and $k$ are colour indices and $\alpha$ is a Dirac index. The quark fields were (Jacobi) smeared to improve the overlap with the $\Lambda$ state.

As usual, the bare matrix elements are determined from ratios of three-point functions and two-point functions

$$
R_{\mathcal{O}}=\frac{\tilde{\Gamma}_{\beta \alpha}\left\langle\Lambda_{\alpha}(t) \mathcal{O}(\tau) \bar{\Lambda}_{\beta}(0)\right\rangle}{\Gamma_{\beta \alpha}\left\langle\Lambda_{\alpha}(t) \bar{\Lambda}_{\beta}(0)\right\rangle} \quad, \quad 0<\tau<t
$$

with appropriate spin projection matrices $\Gamma$ and $\tilde{\Gamma}$. The choice for $\Gamma$ and $\tilde{\Gamma}$ as well as $t$ and the smearing parameters can be found in [12]. The quality of the data can be judged by how well pronounced a plateau is obtained for this ratio as function of $\tau$.

On the lattice the choice of the operators $\mathcal{O}$ is a non-trivial task, because the discretisation reduces the symmetry group of (Euclidean) space-time from $O(4)$ to the hypercubic group $H(4) \subset O(4)$. Therefore, one has to find combinations of operators which avoid the problem of operator mixing [13]. After renormalisation, the matrix elements of these operators are expressed in terms of the reduced matrix elements $v_{2}, a_{0}, a_{1}$ and $t_{0}$ which correspond to moments of the parton distribution functions. We work with the operators

$$
\begin{gathered}
\mathcal{O}_{v_{2}}=\frac{1}{2} \bar{q}\left(\gamma_{4} \stackrel{\leftrightarrow}{D}_{4}-\frac{1}{3}\left(\gamma_{1} \stackrel{\leftrightarrow}{D}_{1}+\gamma_{2} \stackrel{\leftrightarrow}{D}_{2}+\gamma_{3} \stackrel{\leftrightarrow}{D}_{3}\right)\right) q \\
\mathcal{O}_{a_{0}}=\bar{q} \gamma_{2} \gamma_{5} q \\
\mathcal{O}_{a_{1}}=\frac{1}{4} \bar{q}\left(\gamma_{4} \gamma_{5} \stackrel{\leftrightarrow}{D}_{2}+\gamma_{2} \gamma_{5} \stackrel{\leftrightarrow}{D}_{4}\right) q \\
\mathcal{O}_{t_{0}}=\bar{q} \sigma_{24} \gamma_{5} q
\end{gathered}
$$


While the $v_{2}$ operator Eq.(6) (the $v_{2 b}$ operator in the notation of [12]) is used in a spin-averaged matrix element, the other operators require a polarised baryon state to give a non-zero result. In these latter cases we choose the spin to point into the 2-direction. In parton model language we have:

$$
v_{2}^{q}=\langle x q(x)\rangle, \quad a_{0}^{q}=2\langle\Delta q(x)\rangle \quad, \quad a_{1}^{q}=2\langle x \Delta q(x)\rangle \quad, \quad t_{0}^{q}=2\langle\delta q(x)\rangle .
$$

In order to reduce the cut-off effects from $O(a)$ to $O\left(a^{2}\right)$ also in matrix elements, the improvement of the fermionic action has to be accompanied by the improvement of the operator under study. For the axial vector current the improved renormalised operator has the form

$$
A_{\mu}^{i m p r}=Z_{a_{0}}\left(1+b_{a_{0}} a m\right)\left(A_{\mu}+a c_{a_{0}} \partial_{\mu} P\right)
$$

with the bare axial vector current $A_{\mu}(x)=\bar{q}(x) \gamma_{\mu} \gamma_{5} q(x)$, the pseudoscalar density $P(x)=\bar{q}(x) \gamma_{5} q(x)$, and the bare quark mass am $=1 /(2 \kappa)-1 /\left(2 \kappa_{c}\right)$. The improvement term $\partial_{\mu} P$ vanishes in forward matrix elements, so we do not need the coefficient $c_{a_{0}}$. For the other operators one can use the equation of motion to define the improvement coefficients analogous to $c_{a_{0}}$ such that they are of order $g^{2}$ and and thus small. We therefore decided in the end to neglect the improvement terms altogether. For baryons with momentum zero the ratios $R_{\mathcal{O}}$ are related to the reduced matrix elements (10) by

$$
\begin{aligned}
R_{v_{2}} & =-\frac{1}{Z_{v_{2}}\left(1+b_{v_{2}} a m\right)} \frac{1}{2 \kappa} M v_{2}, \\
R_{a_{0}} & =\frac{\mathrm{i}}{Z_{a_{0}}\left(1+b_{a_{0}} a m\right)} \frac{1}{2 \kappa} \frac{1}{2} a_{0}, \\
R_{a_{1}} & =-\frac{\mathrm{i}}{Z_{a_{1}}\left(1+b_{a_{1}} a m\right)} \frac{1}{2 \kappa} \frac{M}{4} a_{1}, \\
R_{t_{0}} & =\frac{1}{Z_{t_{0}}\left(1+b_{t_{0}} a m\right)} \frac{1}{2 \kappa} \frac{1}{2} t_{0} .
\end{aligned}
$$

Depending on the flavour of the quark one has to insert for $\kappa$ either $\kappa_{d}$ or $\kappa_{s}$. These relations account for the operator renormalisation and different normalisations on the lattice and in the continuum [12, 14]. The renormalisation constants $Z_{v_{2}}, Z_{a_{0}}, Z_{a_{1}}, Z_{t_{0}}$ depend on the renormalisation scale $\mu$ of the continuum theory and the lattice cut-off $1 / a$ or equivalently $\beta$. One exception is $Z_{a_{0}}$, which depends only on $\beta$, because in the continuum the anomalous dimension is zero due to current conservation. The scale dependence can be factorized into a factor depending on $\mu$ and one depending on $\beta$. 
For the renormalisation constants we employ a variation of tadpole improved perturbation theory - TRB-PT - as briefly described in [15. The renormalisation group invariant form is first found (accurate to two loop perturbation theory) and then converted back to the $\overline{M S}$-scheme at $\mu^{2}=4 \mathrm{GeV}^{2}$, using two-loop perturbation theory for $t_{0}$ and three-loop perturbation theory for $a_{1}$ and $v_{2}$. We obtain [16]

$$
\begin{aligned}
Z_{v_{2}}\left(\mu^{2}=4 \mathrm{GeV}^{2}, \beta=6.0\right) & =1.11106 & & b_{v_{2}}=1.25803 \\
Z_{a_{0}}(\beta=6.0) & =0.83232 & & b_{a_{0}}=1.27134 \\
Z_{a_{1}}\left(\mu^{2}=4 \mathrm{GeV}^{2}, \beta=6.0\right) & =1.11800 & & b_{a_{1}}=1.24313 \\
Z_{t_{0}}\left(\mu^{2}=4 \mathrm{GeV}^{2}, \beta=6.0\right) & =0.88924 & & b_{t_{0}}=1.24626
\end{aligned} .
$$

$Z_{a_{0}}$ and $b_{a_{0}}$ have been determined non-perturbatively (on the lattice) in 17 with the result

$$
Z_{a_{0}}(\beta=6.0)=0.807(2)(8) \quad b_{a_{0}}=1.28(3)(4) \quad .
$$

This value for $Z_{a_{0}}$ deviates by $\approx 4 \%$ from the perturbative value in Eq. (13). We used for $a_{0}$ Eq.(14) and otherwise Eq.(13). At this point we also want to address the question with which phenomenological distribution functions we should compare. Our $Z$ values include all contributions of order $\alpha_{s}$ and some (but not all) contributions of higher order. This suggests that we should compare them with an NLO (or higher) DGLAP-fit to the experimental data in the $\overline{M S}$ scheme and for the scale $4 \mathrm{GeV}^{2}$.

\section{RESULTS}

From the $\Lambda$ masses at our nine combinations of $\kappa_{d}, \kappa_{s}$ we have computed $\Lambda$ masses at $\kappa_{d}=\kappa_{c}$ by linear extrapolation of $M_{\Lambda}^{2}$ in $1 / \kappa_{d}$. These 12 masses are plotted in Fig. 11. The filled triangles denote the chiral limit $\kappa_{d}, \kappa_{u} \rightarrow$ $\kappa_{c}$ for each $\kappa_{s}$. Our value for $\kappa_{s}^{*}$, which we fixed by $M_{K}$, reproduces quite accurately the ratio $M_{\Lambda} / M_{p}=1.19$. This fits in nicely with the observation that $M_{\Sigma} / M_{p}$ and $M_{\Xi} / M_{p}$ are also rather well reproduced by quenched simulations (see, e.g., [11]). The plot shows clearly the breaking of the $\mathrm{SU}(3)$ flavour symmetry. In particular, the dependence of the masses on $\kappa_{d}$ is rather pronounced.

In Fig. 2 we plot our bare results, i.e. the results without the factors $Z(1+$ bam $)$ in Eq. (12), for $\langle\Delta q(x)\rangle,\langle x \Delta q(x)\rangle\langle x q(x)\rangle$, and $\langle\delta q(x)\rangle$ in the 


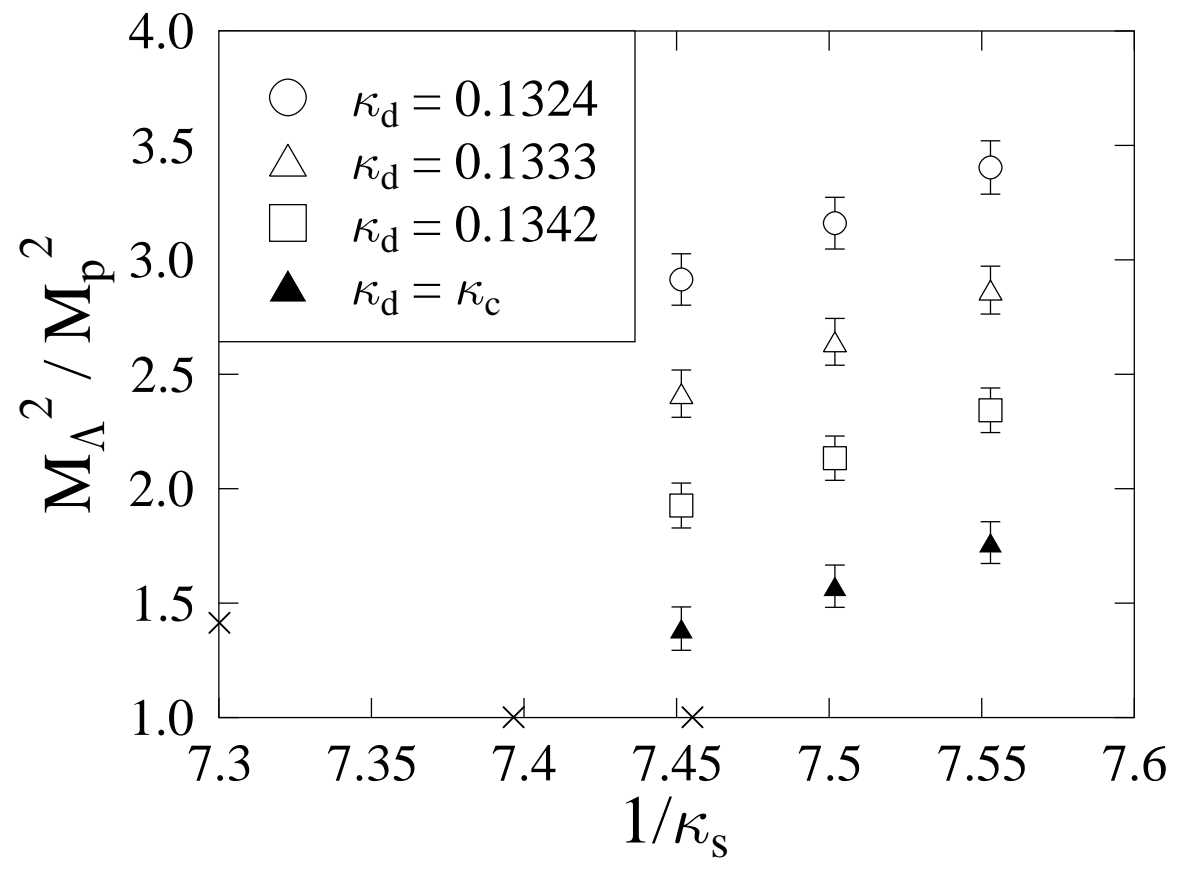

Figure 1: The square of the ratio $M_{\Lambda} / M_{p}$ with the proton mass $M_{p}$ taken in the chiral limit versus $1 / \kappa_{s}$. The different symbols correspond to the different values of $\kappa_{d}$ including the chiral limit. The crosses (left to right) indicate the physical value of $M_{\Lambda} / M_{p}, 1 / \kappa_{c}$, and $1 / \kappa_{s}^{*}$, respectively. 


\begin{tabular}{lcc}
\hline & $u, d$ & $s$ \\
\hline$\langle x q(x)\rangle$ & $0.20(1)$ & $0.27(1)$ \\
$\langle\Delta q(x)\rangle$ & $-0.02(4)$ & $0.68(4)$ \\
$\langle x \Delta q(x)\rangle$ & $0.033(8)$ & $0.23(2)$ \\
$\langle\delta q(x)\rangle$ & $0.03(2)$ & $0.74(4)$ \\
\hline
\end{tabular}

Table 1: Our results for different moments of polarised and unpolarised quark distribution functions in the $\overline{M S}$ scheme at $\mu^{2}=4 \mathrm{GeV}^{2}$.

$\Lambda$ versus $1 / \kappa_{s}(q=s, d$; the results for $u$ are the same as for $d)$. We plotted the bare results because these are the numbers we actually used for interand extrapolation. In contrast with the case of $M_{\Lambda} / M_{p}$, the dependence on $\kappa_{d}$ is rather weak. The values corresponding to $\kappa_{d}=\kappa_{c}$ have been obtained by extrapolating the bare matrix elements linearly in $1 / \kappa_{d}$ (filled triangles). Finally, by interpolating the bare matrix elements for $\kappa_{d}=\kappa_{c}$ linearly in $1 / \kappa_{s}$ to $1 / \kappa_{s}^{*}$ we obtain the desired $\Lambda$ matrix elements. The numbers plotted in the figure are given as tables in the appendix. The physical results for the renormalisation scale $\mu^{2}=4 \mathrm{GeV}^{2}$ are collected in table [1. Thus we find that the $d$ and $u$ quarks carry a somewhat smaller momentum fraction than the $s$ quarks, $20 \%$ each as compared to $27 \%$ which is quite intuitive in view of the larger $s$ mass. We also find that the tensor charge $\delta q$ is close to $\Delta q$ for the $s$ quark, which again fits to our understanding of transversity, as both should be identical in the non-relativistic limit. Note that a positive value of $\langle x \Delta d(x)\rangle$ and a smaller or even negative value for $\langle\Delta d(x)\rangle$ imply a sign change of $\Delta d(x)$ as function of $x$. This would agree with model predictions [1].

These numbers are subject to systematic uncertainties which we will now discuss. For the nucleon similar quenched lattice calculations always gave quark momentum fractions which were too large by typically about $20 \%$ and this disagreement was usually attributed to quenching and the neglect of quark-line disconnected contributions. Recently it has been pointed out that the extrapolation to the chiral limit could be of similar or even greater importance [18]. If this is true we expect that the corrections from a nontrivial chiral extrapolation can affect substantially the values for $\Delta q$. Another source of uncertainty is the lacking continuum extrapolation as the current calculations have been performed at one lattice spacing only.

Let us now discuss our results for $\Delta q$ in more detail on the basis of table 2 . 

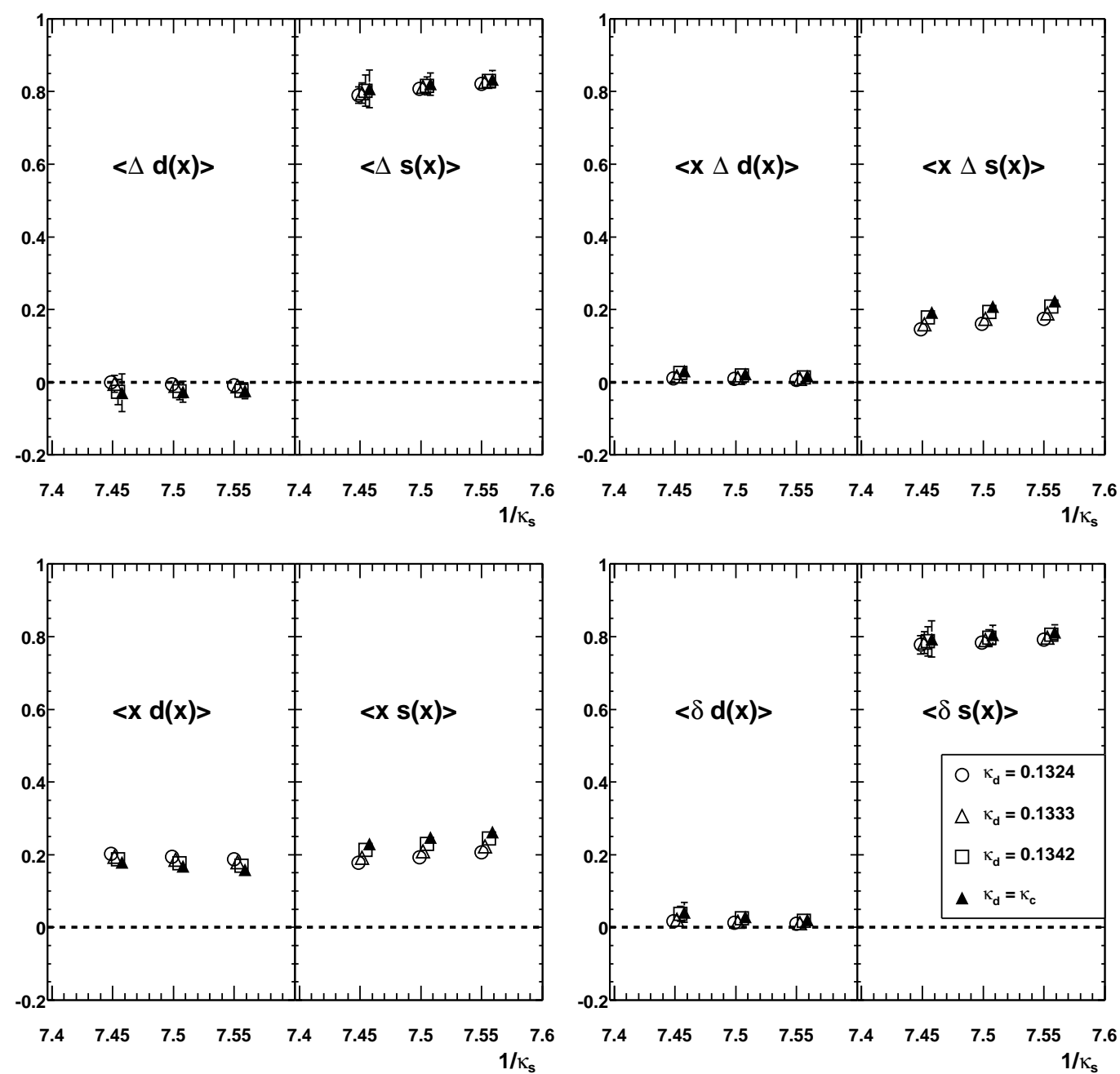

Figure 2: The bare results for $\langle\Delta q(x)\rangle,\langle x \Delta q(x)\rangle,\langle x q(x)\rangle$, and $\langle\delta q(x)\rangle$ in the $\Lambda$ versus $1 / \kappa_{s}$. The results for the different values of $\kappa_{d} \neq \kappa_{c}$ have been slightly displaced horizontally. 


\begin{tabular}{lcc}
\hline & $\Delta u_{\Lambda}=\Delta d_{\Lambda}$ & $\Delta s_{\Lambda}$ \\
\hline quark model & 0 & 1 \\
exp. $+\mathrm{SU}(3)_{\mathrm{F}}$ & $-0.17(3)$ & $0.63(3)$ \\
$\mathrm{MC}+\mathrm{SU}(3)_{\mathrm{F}}$ & $-0.016(9)$ & $0.65(2)$ \\
this work & $-0.02(4)$ & $0.68(4)$ \\
\hline
\end{tabular}

Table 2: Comparison of our results for the longitudinal quark polarisation in the $\Lambda$ with the naive quark model prediction, the flavour SU(3) rotated experimental values for the proton and flavour rotated lattice results for the proton.

In this table we give in the third line the $\Lambda$ matrix elements as they follow from our Monte Carlo results for the proton matrix elements by the use of $\mathrm{SU}(3)_{\mathrm{F}}$ (see Eq.(3)). They agree quite well with the matrix elements computed directly (fourth line). This implies that the flavour symmetry breaking effects in the matrix elements are rather small, which can also be concluded from Fig. 2. As the mass difference between the light quarks and the strange quark was consistently taken into account this provides a strong argument that the $\Lambda$ and proton spin structures are, in good approximation, simply related by an $\mathrm{SU}(3)$ transformation. Consequently, the values given in the second line, which were computed from the proton spin structure under the assumption of flavour SU(3)(see, e.g., [5]), should be quite reliable.

Because we performed a quenched calculation it might be more consistent to compare our results to the prediction for the valence quark contribution, e.g. by Ashery and Lipkin [5]. They obtained $\Delta u_{\Lambda}=\Delta d_{\Lambda}=-0.07(4)$, $\Delta s_{\Lambda}=0.73(4)$. Notice that all results differ markedly from the predictions of the (naive) quark model shown in the first line.

\section{SUMMARY}

We have studied various aspects of the $\Lambda$ (spin) structure taking the mass difference between the quarks into account. We found that our results agree nicely with general expectations: The momentum fraction carried by the $s$ quark is larger than for light quarks, the values for the tensor charge $\delta s$ and $\Delta s$ are similar. Our main result is, however, that $\mathrm{SU}(3)$ flavour symmetry 
appears to be much less violated in the matrix elements than in the baryon masses, which is in agreement with the empirical observation that the hyperon semileptonic decays can be parametrised rather well assuming $\mathrm{SU}(3)$ flavour symmetry. Presently the use of $\Lambda \mathrm{s}$ as a probe of the nucleon spin structure in e.g. deep-inelastic lepton-nucleon scattering still has to rely on models, but the properties of the latter should definitely be checked by a comparison with our lattice results.

We expect that our results have a similar uncertainty as those for the (spin) structure of the proton and that the origin of this uncertainty is the same. When configurations with lighter dynamical quarks become available and our understanding of chiral extrapolation improves, high precision results for the $\Lambda$ should become reachable along the lines discussed in this contribution.

\section{ACKNOWLEDGEMENTS}

This work is supported in part by the DFG (Schwerpunkt "Elektromagnetische Sonden") by BMBF and by the European Community's Human Potential Program under contract HPRN-CT-2000-00145, Hadrons/Lattice QCD. The numerical calculations were performed on the Quadrics computers at DESY Zeuthen. We wish to thank the operating staff for their support.

\section{APPENDIX}

The following tables contain the numerical values for our lattice results. 


\begin{tabular}{ll||ll||ll}
$\kappa_{d}$ & $\kappa_{s}$ & $a_{0}^{d}$ & $a_{0}^{s}$ & $a_{1}^{d}$ & $a_{1}^{s}$ \\
\hline \hline 0.1324 & 0.1324 & $-0.02(1)$ & $1.64(2)$ & $0.014(4)$ & $0.350(7)$ \\
0.1333 & 0.1324 & $-0.02(2)$ & $1.65(3)$ & $0.019(5)$ & $0.378(9)$ \\
0.1342 & 0.1324 & $-0.05(4)$ & $1.66(4)$ & $0.027(7)$ & $0.416(15)$ \\
0.1324 & 0.1333 & $-0.01(2)$ & $1.61(3)$ & $0.017(4)$ & $0.321(8)$ \\
0.1333 & 0.1333 & $-0.02(3)$ & $1.63(3)$ & $0.024(6)$ & $0.350(10)$ \\
0.1342 & 0.1333 & $-0.05(5)$ & $1.63(5)$ & $0.037(9)$ & $0.387(18)$ \\
0.1324 & 0.1342 & $-0.00(3)$ & $1.58(4)$ & $0.022(6)$ & $0.291(9)$ \\
0.1333 & 0.1342 & $-0.01(5)$ & $1.59(6)$ & $0.032(7)$ & $0.318(13)$ \\
0.1342 & 0.1342 & $-0.05(7)$ & $1.60(9)$ & $0.051(12)$ & $0.357(27)$ \\
\hline$\kappa_{\mathrm{c}}$ & 0.1324 & $-0.05(4)$ & $1.67(5)$ & $0.03(1)$ & $0.45(2)$ \\
$\kappa_{\mathrm{c}}$ & 0.1333 & $-0.05(6)$ & $1.64(6)$ & $0.05(1)$ & $0.42(2)$ \\
$\kappa_{\mathrm{c}}$ & 0.1342 & $-0.06(10)$ & $1.61(10)$ & $0.06(2)$ & $0.38(3)$ \\
\hline$\kappa_{\mathrm{c}}$ & $\kappa_{s}^{*}$ & $-0.06(9)$ & $1.62(9)$ & $0.06(2)$ & $0.39(3)$
\end{tabular}

Table 3: Measured bare values for $a_{0}$ and $a_{1}$.

\begin{tabular}{ll||ll||ll}
$\kappa_{d}$ & $\kappa_{s}$ & $v_{2}^{d}$ & $v_{2}^{s}$ & $t_{0}^{d}$ & $t_{0}^{s}$ \\
\hline \hline 0.1324 & 0.1324 & $0.188(2)$ & $0.207(3)$ & $0.02(1)$ & $1.58(2)$ \\
0.1333 & 0.1324 & $0.179(3)$ & $0.223(4)$ & $0.02(1)$ & $1.60(3)$ \\
0.1342 & 0.1324 & $0.169(5)$ & $0.244(5)$ & $0.04(2)$ & $1.61(3)$ \\
0.1324 & 0.1333 & $0.194(3)$ & $0.193(4)$ & $0.03(1)$ & $1.57(3)$ \\
0.1333 & 0.1333 & $0.186(4)$ & $0.209(4)$ & $0.03(1)$ & $1.58(3)$ \\
0.1342 & 0.1333 & $0.177(7)$ & $0.230(6)$ & $0.05(3)$ & $1.59(4)$ \\
0.1324 & 0.1342 & $0.202(3)$ & $0.177(5)$ & $0.03(1)$ & $1.55(5)$ \\
0.1333 & 0.1342 & $0.195(4)$ & $0.192(6)$ & $0.04(2)$ & $1.57(6)$ \\
0.1342 & 0.1342 & $0.188(9)$ & $0.213(9)$ & $0.08(4)$ & $1.57(8)$ \\
\hline$\kappa_{\mathrm{c}}$ & 0.1324 & $0.159(7)$ & $0.262(6)$ & $0.04(3)$ & $1.62(4)$ \\
$\kappa_{\mathrm{c}}$ & 0.1333 & $0.168(8)$ & $0.248(7)$ & $0.06(4)$ & $1.61(5)$ \\
$\kappa_{\mathrm{c}}$ & 0.1342 & $0.180(11)$ & $0.229(10)$ & $0.08(5)$ & $1.59(10)$ \\
\hline$\kappa_{\mathrm{c}}$ & $\kappa_{s}^{*}$ & $0.178(10)$ & $0.233(10)$ & $0.08(5)$ & $1.59(8)$
\end{tabular}

Table 4: Measured bare values for $v_{2}$ and $t_{0}$. 


\section{References}

[1] B. Ehrnsperger and A. Schäfer, Phys. Lett. B 348 (1995) 619;

J. Lichtenstadt and H. Lipkin, Phys. Lett. B 353 (1995) 119;

J. Dai, R. F. Dashen, E. Jenkins, and A. V. Manohar, Phys. Rev. D 53 (1996) 273;

M. J. Savage and J. Walden, Phys. Rev. D 55 (1997) 5376;

J. Linde and H. Snellman, Physica Scripta 58 (1998) 102;

D. de Florian, M. Stratmann, and W. Vogelsang, Phys. Rev. D57 (1998)

9 ;

M. Karliner and H.J. Lipkin, Phys. Lett. B 461 (1999) 280;

H. Weigel, Nucl. Phys. A 680 (2000) 48.

[2] H.-C. Kim, M. Praszalowicz, and K. Goeke, 9th International Workshop on High-Energy Spin Physics (SPIN 01), Dubna, Russia, 2001, hepph/0110135.

[3] M. Burkardt and R.L. Jaffe, Phys. Rev. Lett. 70 (1993) 2537, R.L. Jaffe Phys. Rev. D 54 (1996) 6581.

[4] J. R. Ellis, D. Kharzeev, and A. Kotzinian, Z. Phys. C 69 (1996) 467; H.-C. Kim, M. Praszalowicz, and K. Goeke, Acta Phys. Polon. B 31 (2000) 1767;

B.Q. Ma, I. Schmidt, J. Soffer and J.-J. Yang, Phys. Rev. D 62 (2000) 114009; Phys. Rev. D 64 (2001) 014017; Phys. Rev. D 65 (2002) 034004; Phys. Lett. B 488 (2000) 254; Phys. Lett. B 489 (2000) 293;

T. Londergan and A.W. Thomas, Phys. Rev. D 61 (2000) 014007, Phys. Rev. D62 (2000) 014021.

[5] D. Ashery and H.J. Lipkin, Phys. Lett. B 469 (1999) 263.

[6] ALEPH Collaboration, D. Buskulic et al., Phys. Lett. B 374 (1996) 319; OPAL Collaboration, K. Ackerstaff et al., Eur. Phys. J. C 2 (1998) 49.

[7] HERMES Collaboration, A. Airapetian et al., Phys. Rev. D 64 (2001) 112005.

[8] P. Astier et al. (NOMAD), Nucl. Phys. B 605 (2001) 3; Nucl. Phys. B 588 (2000) 3 . 
[9] K. Heller, proceedings of SPIN 96, page 23; C.W. de Jager, T.J. Ketel, P.J. Mulders, J.E.J. Oberski, M. Oskam-Tamboezer (eds.); World Scientific, Singapore, 1997;

J. Lach, Nucl. Phys. (Proc. Suppl) B 50 (1996) 216.

[10] M. Göckeler, R. Horsley, D. Pleiter, P.E.L. Rakow, S. Schaefer, A. Schäfer and G. Schierholz, Nucl. Phys. Proc. Suppl. 106 (2002) 305.

[11] D. Pleiter, Thesis, Berlin (2000); QCDSF Collaboration, in preparation.

[12] M. Göckeler, R. Horsley, E.M. Ilgenfritz, H. Perlt, P. Rakow, G. Schierholz and A. Schiller, Phys. Rev. D 53 (1996) 2317.

[13] G. Martinelli and C.T. Sachrajda, Nucl. Phys. B 306 (1988) 865; M. Göckeler, R. Horsley, E. M. Ilgenfritz, H. Perlt, P. Rakow, G. Schierholz and A. Schiller, Phys. Rev. D 54 (1996) 5705.

[14] M. Göckeler, R. Horsley, L. Mankiewicz, H. Perlt, P. Rakow, G. Schierholz and A. Schiller, Phys. Lett. B 414 (1997) 340.

[15] S. Capitani, M. Göckeler, R. Horsley, D. Pleiter, P. E. L. Rakow, H. Stüben and G. Schierholz, Nucl. Phys. B (Proc. Suppl.) 106 (2002) 299; QCDSF collaboration, in preparation.

[16] S. Capitani, M. Göckeler, R. Horsley, H. Perlt, P. E. L. Rakow, G. Schierholz and A. Schiller, Nucl. Phys. B 593 (2001) 183.

[17] T. Bhattacharya, R. Gupta, W. Lee and S. Sharpe, Phys. Rev. D 63 (2001) 074505.

[18] T. Hemmert and W. Weise, preprint hep-lat/0204005; W. Detmold, W. Melnitchouk, and A.W. Thomas, preprint hep-lat/0206001, and references in both papers. 